\title{
Effect of temperature on the survival and development of the immature stages of Monosteira unicostata (Hemiptera: Tingidae)
}

\author{
Ismael SÁNCHEZ-RAMOS, SusAna PASCUAL, Cristina E. FERNÁNDEZ, ArÁNZAZu MARCOTEGUI \\ and MANUEL GONZÁLEZ-NÚÑEZ
}

\begin{abstract}
Entomology Group, Plant Protection Department, Instituto Nacional de Investigación y Tecnología Agraria y Alimentaria (INIA), Carretera de La Coruña Km 7,5, 28040 Madrid, Spain; e-mails: ismael@inia.es; pascual@inia.es; delaguia@inia.es; marcotegui@gmail.com;mgnunez@inia.es
\end{abstract}

Key words. Hemiptera, Tingidae, Monosteira unicostata, immature survival, immature development, temperature, thermal threshold

\begin{abstract}
The poplar lace bug, Monosteira unicostata (Mulsant \& Rey), is one of the most important pests of almond trees in the Mediterranean area. The developmental times and survival of the immature stages of this tingid were determined at 16, 19, 22, 25, $28,31,34,35.5,37$ and $39^{\circ} \mathrm{C}, 60 \pm 10 \%$ relative humidity and under a $16 \mathrm{~L}: 8 \mathrm{D}$ photoperiod. At 16 and $39^{\circ} \mathrm{C}, 100 \%$ mortality was recorded during immature development, with the egg, first and second nymphal instars the most susceptible. The lowest mortality was recorded at $28^{\circ} \mathrm{C}(9.8 \%)$. As temperature increased, the total developmental period decreased and the shortest duration was recorded at $34^{\circ} \mathrm{C}(12.1-12.2 \mathrm{~d})$. At $37^{\circ} \mathrm{C}$, the developmental time was slightly longer (12.5-13.0 d). The thermal requirement for complete development, from egg to adult, was 229.2 DD. Several non-linear models were fitted to the developmental rate data recorded for the range of temperatures tested. Selection criteria indicated that the Lactin model best described the relationship between developmental rate and temperature. It predicted lower and upper thermal thresholds for the complete preimaginal period of 14.8 and $39.1^{\circ} \mathrm{C}$, respectively. Models were validated by comparing their predictions for the total preimaginal period with the developmental times recorded at three different fluctuating temperature regimes. The Lactin model again best fitted the actual durations recorded. This model could be used in IPM programs for predicting the moment of occurrence of the most damaging generations of this species.
\end{abstract}

\section{INTRODUCTION}

The poplar lace bug, Monosteira unicostata (Mulsant \& Rey), is one of the most important pests of almond trees in the Mediterranean area (Maniglia, 1983; Liotta \& Maniglia, 1994; García Marí \& Ferragut, 2002) and has been detected also in North America (Scudder, 2012). This species has a broad range of host plants and it has been cited attacking other fruit trees such as cherry, peach, plum, pear and forest trees like poplar or willow (Péricart, 1983).

This insect overwinters as an adult under fallen leaves, on the bark of trees or on wild plants (Talhouk, 1977; Liotta \& Maniglia, 1994; García Marí \& Ferragut, 2002), but on warm days in winter adults can leave their winter refuges for a few hours (Vessia, 1961; Maniglia, 1983). The overwintering period depends on how hard the winter is so in zones with mild winters overwintering can be as short as a couple of months (Vidal, 1938). All these observations suggest that during winter these insects are in a state of quiescence. In spring, the adults feed on young almond leaves where the females lay eggs and start infestations (Talhouk, 1977; Liotta \& Maniglia, 1994; García Marí \& Ferragut, 2002). After hatching, nymphs begin to feed on the lower side of the leaves and pass through five instars (Fig. 1) before becoming adults. Nymphs of this species show a sedentary and gregarious behaviour and when disturbed disperse and regroup later (Bremond, 1938; Vessia, 1961; Neal \& Schaefer, 2000). Their feeding activity damages leaves, which weaken, turn yellow and often fall, which reduces the development and maturation of the fruit
(Gómez-Menor, 1950; Liotta \& Maniglia, 1994) and has negative consequences for the following year's production (Liotta \& Maniglia, 1994). In addition, the accumulation of excrement on the leaves reduces gas exchange (Bremond, 1938; Gómez-Menor, 1950) and it is reported that the females in laying eggs inside the parenchyma of the leaves facilitates the infection of plants with fungi and bacteria (Gómez-Menor, 1950).

The number of generations in different geographical areas where this insect occurs varies from 2 to 4 (Péricart, 1983). These generations overlap, so that all immature stages and instars are present simultaneously during the summer (Péricart, 1983). In Spain there are 3-4 generations and the greatest injury is caused by the third generation, when the insects are most numerous (Gómez-Menor, 1950; García Marí \& Ferragut, 2002).

Insects are poikilothermic organisms so temperature is one of the main factors determining their physiological processes and a key factor determining their phenology (Régnière et al., 2012; Régnière \& Powell, 2013). Accordingly, one important aspect of pest management is the prediction of the occurrence of the different life stages based on temperatures recorded in the field (Logan, 1988). This approach is usually based on laboratory experiments in which the durations of the developmental periods of the immature stages is established under a wide range of constant temperatures (Wagner et al., 1984a, b). Then, the developmental rates (the inverse of the developmental periods) are plotted against temperature and fitted by mathe- 

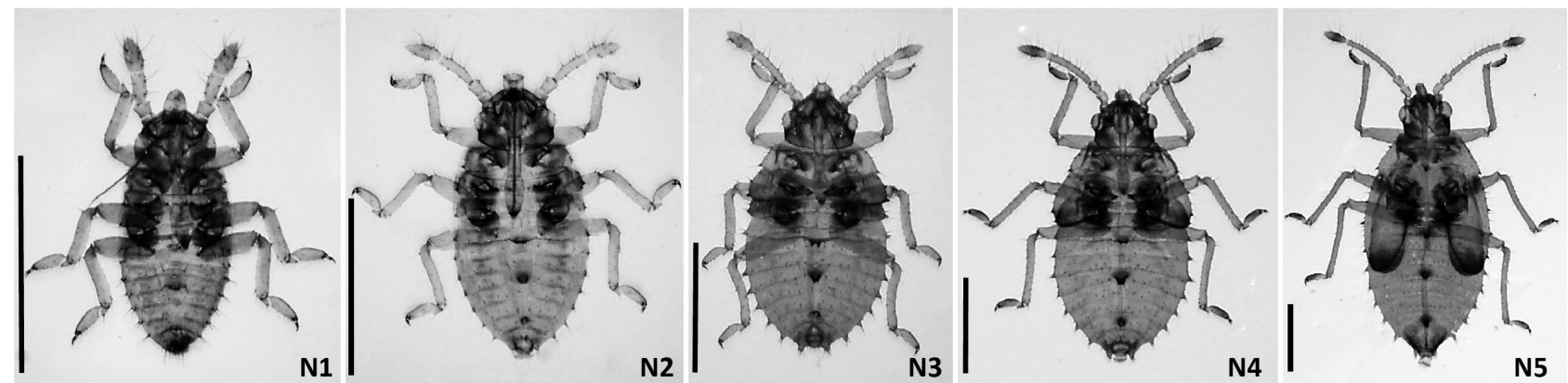

Fig. 1. First (N1), second (N2), third (N3), fourth (N4) and fifth (N5) nymphal instars of Monosteira unicostata. Specimens were mounted on slides in Hoyer's medium after digesting the internal tissues using lactic acid and staining the cuticle. Scale bars correspond to $0.5 \mathrm{~mm}$.

matical functions to obtain reliable models of this relationship, which has a generic canonical form (Wagner et al., 1984a; Logan, 1988). The integration of models obtained under the fluctuating temperature regimes recorded in the field allows the prediction of the occurrence of the different generations or the most damaging stages of pests. For this a variety of functions are used, ranging from the classical linear degree-day model to more complex nonlinear models (Schoolfield et al., 1981; Hilbert \& Logan, 1983; Lactin et al., 1995; Briere et al., 1999; Kontodimas et al., 2004; Hansen et al., 2011; Shi et al., 2011; Régnière et al., 2012). Some of these models can also be used to determine the lower and upper thermal developmental thresholds of the immature stages.

Developmental periods usually vary considerably among individuals in insect populations. To deal with this stochastic aspect of development in a population, different functions have been employed to describe the cumulative probability distribution of insect development in time (Wagner et al., 1984b). Thus, these probability functions can be used to determine the fraction of a cohort that will have completed its development at a particular time under field temperatures.

The aim of the present work was to investigate the effect of temperature on the survival and developmental periods of the immature stages of $M$. unicostata and establish the distribution of adult emergence for this species. Different linear and non-linear models were used to describe the relationship between the developmental parameters of the immature stages and temperature. Also, the developmental models obtained were validated by comparing their predictive capacity with developmental data recorded at fluctuating temperatures.

\section{MATERIAL AND METHODS}

\section{Laboratory rearing of Monosteira unicostata}

A colony of M. unicostata was established in the laboratory from individuals collected in mid spring from almond trees, Prunus dulcis (Mill.) D.A. Webb, placed in INIA (Madrid). The insects were then transferred to the laboratory and reared on small black poplar plants, Populus nigra L., grown from hardwood cuttings, because it is not possible to produce small almond trees in this way. The $15-20 \mathrm{~cm}$ long cuttings were collected in winter and kept refrigerated at $4^{\circ} \mathrm{C}$. When needed, they were planted and grown at $25^{\circ} \mathrm{C}, 70 \%$ relative humidity $(\mathrm{RH})$ and under a $16 \mathrm{~L}: 8 \mathrm{D}$ photoperiod until big enough (about $60 \mathrm{~cm}$ height) to be infested. Fifty to sixty adults of $M$. unicostata were used to infest four poplar plants. The infested plants were confined in wooden framed cages $(60 \mathrm{~cm} \times 50 \mathrm{~cm} \times 45 \mathrm{~cm})$ whose sides were covered with translucent nylon cloth. The frontal piece of nylon cloth was removable so that the insects and plants were easily accessible. To maintain the M. unicostata population, new poplar plants were grown and periodically infested with adults from the laboratory rearing when the older plants were seriously damaged.

\section{Plant material}

Assays were performed using small rooted cuttings of black poplar. Poplar twigs were obtained in spring from poplars growing in the surroundings of the INIA facilities. Once in the laboratory, the twigs were washed and inspected and those with symptoms of infestation, disease or any type of physiological damage were discarded. Then, they were cut into smaller cuttings with a single leaf and a small portion of stem. These cuttings were placed in nutritive solution (Moutous \& Fos, 1973) for a week and those that rooted were selected to be used in the developmental assays, thus assuring that the leaves remained alive during the whole experimental period. The rooted cuttings with leaves were introduced into plastic containers $(7 \mathrm{~cm} \mathrm{high} \times 3.5 \mathrm{~cm}$ diameter $)$ containing nutritive solution through a small hole $(8 \mathrm{~mm}$ diameter) in the lid. The hole was then sealed with plasticine.

\section{Immature development}

Development was monitored at constant temperatures of 16,19 , $22,25,28,31,34,35.5,37$ and $39^{\circ} \mathrm{C}$ in environmental chambers (Sanyo MLR-350, Sanyo, Japan). At all temperatures the other environmental conditions were $60 \pm 10 \% \mathrm{RH}$ and a $16 \mathrm{~L}: 8 \mathrm{D}$ photoperiod. Lace bug subpopulations were acclimatized for at least one month at each temperature.

Assays were carried out in rearing units consisting of plastic tubes $(15 \mathrm{~cm}$ high $\times 5.5 \mathrm{~cm}$ diameter $)$ placed vertically in the upper half of a 5.8 diameter Petri dish with the upper opening covered by a piece of translucent nylon cloth held in place by a rubber band, which provided ventilation for the rearing cell.

A container with a rooted cutting with a leaf, as described above, was introduced intro each rearing unit together with 2-3 acclimatized adult pairs of $M$. unicostata. Females were allowed to lay eggs on the leaves for $24 \mathrm{~h}$, and after that all the adults were removed. Then, the leaves were inspected under a stereomicroscope and all the eggs laid during the $24 \mathrm{~h}$ period located. A single egg was randomly selected and the rest killed by puncturing them with a fine needle. 65 to 200 leaves, each with a single egg, were kept at each temperature. Hatching date, duration of each nymphal instar and survival were recorded daily until each individual reached the adult stage or died. The adults were sexed according to Vessia (1961). 
TABLE 1. Mathematical models used to describe the relationship between the developmental rate of the immature stages and instars of M. unicostata and temperature.

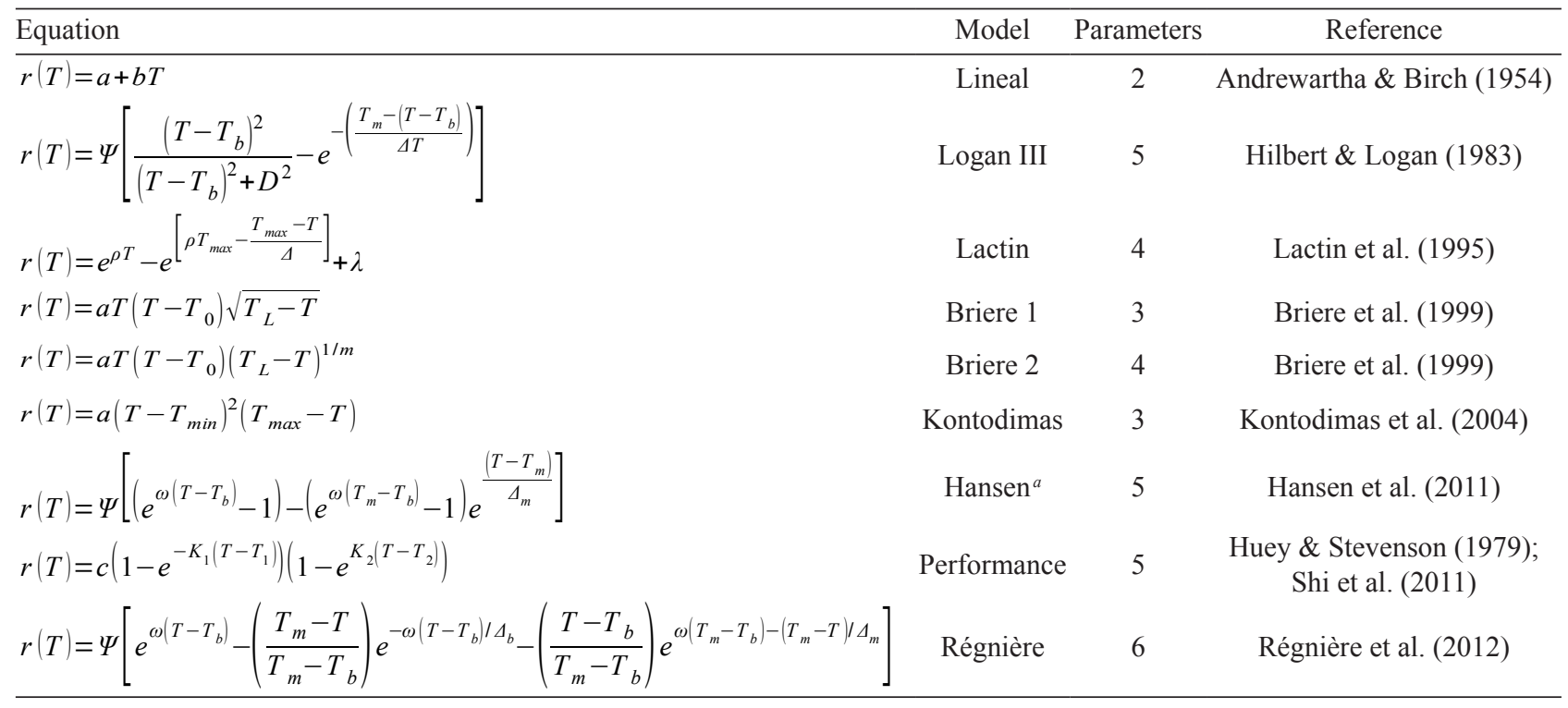

See the corresponding references for a complete description of these models and their parameters. ${ }^{a}$ The description and terminology given by Régnière et al. (2012) is used for the Hansen model.

\section{Mathematical models}

Mortality model

The percentage mortality recorded at each constant temperature for the different immature stages of M. unicostata were fitted by the following equation

$$
y=e^{a+b T+c T^{2}}
$$

where $y$ is the percentage mortality, $T$ temperature and $a, b$ and $c$ are fitted parameters. This model was fitted only to those stages that suffered significant levels of mortality.

Developmental models

The relationship between developmental rate $(r=1 / d$, where $\mathrm{d}$ is the mean developmental time in days) and temperature $\mathrm{T}$, was determined using both linear and nonlinear models (Table 1). This was done for the development of the egg, each of the five nymphal instars and the complete preimaginal period.

In the case of the linear model, the lower developmental threshold was estimated as $L T=-a / b$ and the thermal constant as $K=$ $1 / b$ (Trudgill et al., 2005). The standard errors of $L T$ and $K$ were estimated according to Campbell et al. (1974). To achieve satisfactory fit when using the linear model, only data below $34^{\circ} \mathrm{C}$ were used because if developmental rates for temperatures above this temperature were included the relationship was nonlinear (De Clercq \& Degheele, 1992).

The nonlinear models selected are unlike other nonlinear models in that they can be used to predict both the lower and upper temperature developmental thresholds and the optimal temperature for development (temperature at which developmental time is shortest). In the case of the Logan III and Lactin functions, the thermal thresholds were estimated using the lower and upper intersections of the curve with the $\mathrm{x}$ axis after graphical representation of the models, because none of the parameters in these functions correspond to these temperature values (Hilbert \& Logan, 1983; Lactin et al., 1995). For the other models, the thermal thresholds were estimated using their corresponding parameters. The optimal temperature for development was estimated graphically in all cases as the temperature at which the highest rate of development was predicted.

\section{Model for distribution of adult emergence}

The distribution of adult emergence was determined by calculating the cumulative proportion of individuals that reached the adult stage at normalized time $x=$ time/mean time for each temperature. The relationship was described using the equation of Stinner et al. (1975):

$$
F(z)=(1-z)^{\left(k z^{\beta}\right)}
$$

where $F(z)$ is the cumulative proportion of individuals that completed development, $z=\left(x_{\max }-x\right) /\left(x_{\max }-x_{\min }\right), x$ is normalized time (time/mean time), $x_{\min }$ is normalized time at which the first individual completed development, $x_{\max }$ is normalized time at which the last individual completed development, and $k$ and $\beta$ are empirical constants.

\section{Validation of developmental models}

To validate the developmental models the total development period from egg to adult of $M$. unicostata was evaluated under the following three fluctuating temperature regimes in the sense described by Liu et al. (1995): $28-14^{\circ} \mathrm{C}, 33-18^{\circ} \mathrm{C}$ and $38-22^{\circ} \mathrm{C}$. Within each regime, the upper temperature was associated with the $16 \mathrm{~h}$ of daylight and lower temperature with the $8 \mathrm{~h}$ of night. The experimental procedures were similar to those described above for the evaluation of developmental times at constant temperatures. To obtain the eggs, one female per plant was allowed to lay eggs for $24 \mathrm{~h}$ at $26^{\circ} \mathrm{C}$, but in this case all the eggs laid were used. For further calculations, eggs were considered to be laid at the mid-point of the $24 \mathrm{~h}$ period of egg laying. Afterwards, the rearing units containing the leaves with the eggs were transferred to an environmental chamber (IBERCEX, Spain) and kept at a particular regime of fluctuating temperatures. A data logger ELUSB-2 (Lascar electronics, UK) was put beside the rearing units to record the temperature at $5 \mathrm{~min}$ intervals. Then, an integration of the increments in development for each 5-min interval according to the different models obtained for the complete developmental time was used.

\section{Analytical methods}

Chi-squared tests were performed to assess the effect of temperature on the mortality of the egg and five nymphal instars and 
A

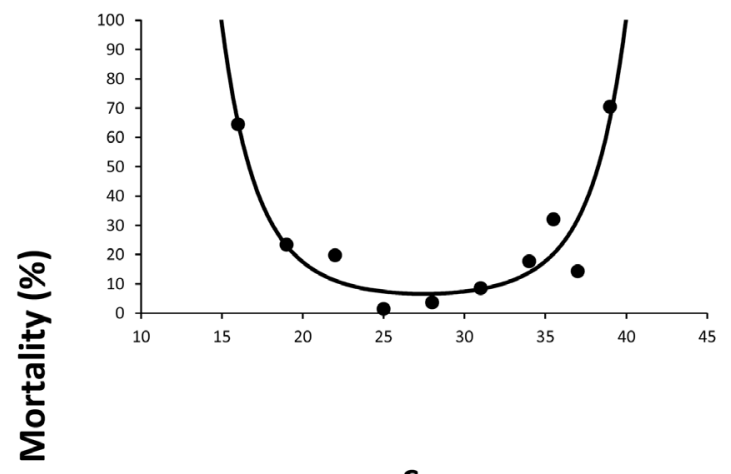

C

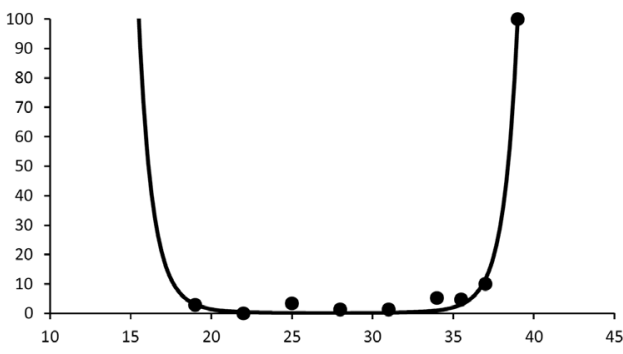

B

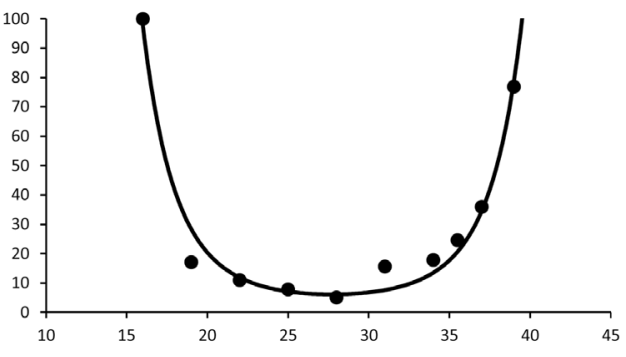

D

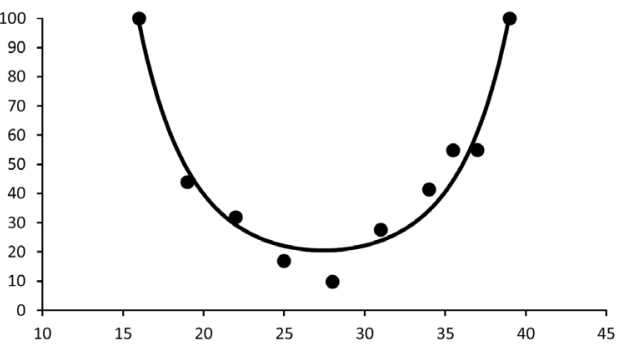

Temperature (이

Fig. 2. Percentage mortality of egg (A), first nymphal instar (B), second nymphal instar (C) and total preimaginal period (D) of Monosteira unicostata recorded at different temperatures. Closed circles are the recorded percentages of mortality and solid lines the fitted curves according to the function $y=e^{a+b T+c T^{2}}$.

the sex ratio of $M$. unicostata. Also, a chi-squared test was used to test for deviations in the expected $1: 1$ sex ratio recorded at each temperature. The effect of temperature on the proportion of the time spent in each developmental stage/instar was tested using a Kruskal-Wallis test separately for each stage/instar. An ANCOVA was used to assess the differences between sexes in the total developmental time, with sex as a fixed factor and temperature a covariate. The level of significance was $P<0.05$ in all cases. Analyses were done using Statgraphics ${ }^{\circledR}$ Centurion XVI (StatPoint Technologies, 2009).

Regarding the modelling of development, the linear model was fitted and parameter values estimated by means of least-squares regression, whereas the Levenburg-Marquardt algorithm was used for nonlinear models. All models were obtained using Tablecurve 2D 5.01 (SYSTAT, 2002). Performance of non-linear developmental models were compared using the adjusted coefficient of determination $\left(R_{a}^{2}\right)$ (Kvalseth, 1985), the Bayesian Information Criterion (BIC) (Schwarz, 1978) and the corrected Akaike Information Criterion $(A I C C)$ (Hurvich \& Tsai, 1989; Burnham $\&$ Anderson, 2002). These fitting criteria allow the comparison of functions with different numbers of parameters. Their expressions are

$$
R_{a}^{2}=1-\left(1-R_{i}^{2}\right)(n-1) /(n-k-1)
$$

where $n$ is the number of observations, $k$ is the number of parameters in the $i t h$ function, and $R_{i}^{2}$ is computed as

$$
R_{i}^{2}=1-\sum_{j=1}^{n}\left(y_{j}-\hat{y}_{j i}\right)^{2} / \sum_{j=1}^{n}\left(y_{j}-\bar{y}\right)^{2}
$$

for $y_{j}$ the $j$ th recorded developmental rate, $\hat{y}_{j i}$ the $j$ th predicted developmental rate from the ith function and $\bar{y}$ the mean of the recorded data (Kvalseth, 1985).

$$
\begin{gathered}
B I C=n \ln (R S S / n)+K \ln (n) \\
A I C c=n \ln (R S S / n)+2 \mathrm{~K}+\frac{2 \mathrm{~K}(K+1)}{n-K-1}
\end{gathered}
$$

where $n$ is the number of observations, $K$ the number of parameters including the error term and RSS the residual sum of squares (Angilletta Jr., 2006; Shi \& Ge, 2010).

TABLE 2. Parameter estimates and coefficients of determination $\left(R^{2}\right)$ for the function $y=e^{a+b T+c T^{2}}$ describing the relationship between mortality during the egg, nymph 1 , nymph 2 and complete preimaginal period and temperature $\left({ }^{\circ} \mathrm{C}\right)$ for $M$. unicostata.

\begin{tabular}{lcccc}
\hline \multirow{2}{*}{ Parameter } & \multicolumn{4}{c}{ Stage / Instar } \\
\cline { 2 - 4 } & Egg & Nymph 1 & Nymph 2 & Egg to adult \\
\hline$a$ & $14.9916(1.9808)$ & $17.3048(1.1449)$ & $34.9315(5.2119)$ & $12.0264(0.7195)$ \\
$b$ & $-0.9536(0.1706)$ & $-1.1177(0.0997)$ & $-2.7326(0.3607)$ & $-0.6562(0.0604)$ \\
$c$ & $0.0174(0.0031)$ & $0.0202(0.0018)$ & $0.0501(0.0059)$ & $0.0120(0.0011)$ \\
$R^{2}$ & 0.8829 & 0.9738 & 0.9956 & 0.9561 \\
\hline
\end{tabular}

Values in parentheses are standard errors. 
TABLE 3. Mean developmental time in days (mean $\pm \mathrm{SE}$ ) of $M$. unicostata immature stages and instars recorded at constant temperatures.

\begin{tabular}{|c|c|c|c|c|c|c|c|c|c|}
\hline \multirow{2}{*}{$\begin{array}{l}\text { Temperature } \\
\left({ }^{\circ} \mathrm{C}\right)\end{array}$} & \multirow{2}{*}{$\begin{array}{c}\text { Initial } \\
\mathrm{n}\end{array}$} & \multicolumn{8}{|c|}{ Stage / Instar (n) } \\
\hline & & Egg & Nymph 1 & Nymph 2 & Nymph 3 & Nymph 4 & Nymph 5 & $\begin{array}{l}\text { Egg to adult } \\
\text { (male) }\end{array}$ & $\begin{array}{l}\text { Egg to adult } \\
\text { (female) }\end{array}$ \\
\hline 16 & 141 & $\begin{array}{c}43.5 \pm 0.5 \\
(50)\end{array}$ & - & - & - & - & - & - & - \\
\hline 19 & 107 & $\begin{array}{l}24.0 \pm 0.3 \\
\quad(82)\end{array}$ & $\begin{array}{l}5.5 \pm 0.1 \\
\quad(68)\end{array}$ & $\begin{array}{l}4.2 \pm 0.1 \\
\quad(66)\end{array}$ & $\begin{array}{c}4.2 \pm 0.1 \\
\quad(64)\end{array}$ & $\begin{array}{c}4.5 \pm 0.1 \\
(61)\end{array}$ & $\begin{array}{c}7.0 \pm 0.1 \\
(60)\end{array}$ & $\begin{array}{l}49.1 \pm 0.7 \\
\quad(27)\end{array}$ & $\begin{array}{l}49.3 \pm 0.8 \\
(33)\end{array}$ \\
\hline 22 & 91 & $\begin{array}{l}14.3 \pm 0.1 \\
(73)\end{array}$ & $\begin{array}{c}3.4 \pm 0.1 \\
(65)\end{array}$ & $\begin{array}{l}2.5 \pm 0.1 \\
(65)\end{array}$ & $\begin{array}{c}2.6 \pm 0.1 \\
(65)\end{array}$ & $\begin{array}{l}2.9 \pm 0.1 \\
(63)\end{array}$ & $\begin{array}{c}4.3 \pm 0.1 \\
(62)\end{array}$ & $\begin{array}{c}30.0 \pm 0.3 \\
(32)\end{array}$ & $\begin{array}{l}30.1 \pm 0.3 \\
\quad(30)\end{array}$ \\
\hline 25 & 65 & $\begin{array}{l}10.4 \pm 0.1 \\
\quad(64)\end{array}$ & $\begin{array}{l}2.7 \pm 0.1 \\
(59)\end{array}$ & $\begin{array}{c}1.9 \pm 0.1 \\
(57)\end{array}$ & $\begin{array}{c}1.9 \pm 0.1 \\
(57)\end{array}$ & $\begin{array}{c}2.0 \pm 0.1 \\
(57)\end{array}$ & $\begin{array}{c}3.3 \pm 0.1 \\
(54)\end{array}$ & $\begin{array}{l}21.9 \pm 0.2 \\
(28)\end{array}$ & $\begin{array}{l}22.3 \pm 0.1 \\
(26)\end{array}$ \\
\hline 28 & 82 & $\begin{array}{c}8.7 \pm 0.1 \\
\quad(79)\end{array}$ & $\begin{array}{c}2.1 \pm 0.1 \\
\quad(75)\end{array}$ & $\begin{array}{c}1.6 \pm 0.1 \\
(74)\end{array}$ & $\begin{array}{c}1.4 \pm 0.1 \\
(74)\end{array}$ & $\begin{array}{c}1.8 \pm 0.1 \\
(74)\end{array}$ & $\begin{array}{l}2.7 \pm 0.1 \\
\quad(74)\end{array}$ & $\begin{array}{l}18.6 \pm 0.2 \\
\quad(27)\end{array}$ & $\begin{array}{l}18.3 \pm 0.2 \\
\quad(47)\end{array}$ \\
\hline 31 & 105 & $\begin{array}{c}6.5 \pm 0.1 \\
(96)\end{array}$ & $\begin{array}{c}1.8 \pm 0.1 \\
(81)\end{array}$ & $\begin{array}{c}1.3 \pm 0.1 \\
(80)\end{array}$ & $\begin{array}{c}1.3 \pm 0.1 \\
(79)\end{array}$ & $\begin{array}{c}1.4 \pm 0.1 \\
(78)\end{array}$ & $\begin{array}{c}2.1 \pm 0.1 \\
(76)\end{array}$ & $\begin{array}{l}14.4 \pm 0.1 \\
(36)\end{array}$ & $\begin{array}{l}14.2 \pm 0.1 \\
\quad(40)\end{array}$ \\
\hline 34 & 198 & $\begin{array}{l}5.7 \pm 0.1 \\
(163)\end{array}$ & $\begin{array}{c}1.6 \pm 0.1 \\
(134)\end{array}$ & $\begin{array}{l}1.1 \pm 0.1 \\
\quad(127)\end{array}$ & $\begin{array}{c}1.0 \pm 0.1 \\
\quad(124)\end{array}$ & $\begin{array}{c}1.1 \pm 0.1 \\
(119)\end{array}$ & $\begin{array}{l}1.8 \pm 0.1 \\
(116)\end{array}$ & $\begin{array}{l}12.2 \pm 0.1 \\
\quad(58)\end{array}$ & $\begin{array}{l}12.1 \pm 0.1 \\
\quad(58)\end{array}$ \\
\hline 35.5 & 84 & $\begin{array}{c}5.8 \pm 0.1 \\
(57)\end{array}$ & $\begin{array}{c}1.5 \pm 0.1 \\
(43)\end{array}$ & $\begin{array}{c}1.0 \pm 0.1 \\
(41)\end{array}$ & $\begin{array}{c}1.0 \pm 0.1 \\
(40)\end{array}$ & $\begin{array}{c}1.2 \pm 0.1 \\
(39)\end{array}$ & $\begin{array}{c}1.8 \pm 0.1 \\
(38)\end{array}$ & $\begin{array}{l}12.4 \pm 0.2 \\
\quad(17)\end{array}$ & $\begin{array}{l}12.4 \pm 0.2 \\
\quad(21)\end{array}$ \\
\hline 37 & 91 & $\begin{array}{l}6.0 \pm 0.1 \\
\quad(78)\end{array}$ & $\begin{array}{l}1.4 \pm 0.1 \\
(50)\end{array}$ & $\begin{array}{c}1.2 \pm 0.1 \\
(45)\end{array}$ & $\begin{array}{c}1.1 \pm 0.1 \\
(42)\end{array}$ & $\begin{array}{c}1.2 \pm 0.1 \\
(41)\end{array}$ & $\begin{array}{c}1.9 \pm 0.1 \\
(41)\end{array}$ & $\begin{array}{c}13.0 \pm 0.2 \\
(26)\end{array}$ & $\begin{array}{c}12.5 \pm 0.2 \\
(15)\end{array}$ \\
\hline 39 & 88 & $\begin{array}{c}6.6 \pm 0.1 \\
(26)\end{array}$ & $\begin{array}{l}2.0 \pm 0.3 \\
(6)\end{array}$ & - & - & - & - & - & - \\
\hline
\end{tabular}

$\mathrm{n}$ - number of individuals at each developmental stage/instar.

In addition, the $z$ weight described by Shi \& Ge (2010) was calculated, which integrates the $R_{a}^{2}, B I C$ and $A I C c$ values of the different models. The $z$ weight is calculated as

$$
z_{i}=D_{i} / \sum_{i=1}^{s} D_{i}
$$

where $D_{i}$ is the weighted mean of standardized indicators in the $i$ th function and $S$ is the number of candidate models. $D_{i}$ is computed as

$D_{i}=\frac{1}{3}\left(\frac{\left|R_{a i}^{2}-\min \left(R_{a}^{2}\right)\right|}{\max \left(R_{a}^{2}\right)-\min \left(R_{a}^{2}\right)}+\frac{\left|B I C_{i}-\max (B I C)\right|}{\max (B I C)-\min (B I C)}+\frac{\left|A I C c_{i}-\max (A I C C)\right|}{\max (A I C c)-\min (A I C c)}\right)$

where $\max (x)$ denotes the maximum $x$ value in all $S$ candidate models, $\min (x)$ denotes the minimum $x$ value in all $S$ candidate models and $x_{i}$ denotes the $x$ value of the ith candidate model. Unlike the original paper by Shi \& Ge (2010), we did not use $R^{2}$ or $R S S$ because these criteria are not corrected for the number of parameters in the models (Angilletta Jr., 2006). In addition, AICC is preferred to $A I C$ when only small sample sizes are available (Burnham \& Anderson, 2002).

Lowest $B I C$ and $A I C C$ and highest $R_{a}^{2}$ and $z$ weight values indicate the best fitting model. In the case of the linear model only $R_{a}^{2}$ was calculated because information criteria can be used only to compare models fitted to exactly the same set of data (Burnham $\&$ Anderson, 2002) and, as stated above, results for temperatures above $34^{\circ} \mathrm{C}$ were not included in the linear model.

The total preimaginal periods predicted by the models for the three fluctuating temperature regimes were compared with the actual durations of development. The adjustment was expressed as the percentage deviation with regard to the recorded developmental times, using to the following expression (Liu et al., 1995):

$$
d=\left[\frac{P D T}{R D T}-1\right] \times 100
$$

where $d$ is the percentage deviation, PDT is the predicted developmental time for each function and $R D T$ is the recorded developmental time. Negative values indicate under estimate of the developmental time and positive values indicate an over estimate.

\section{RESULTS}

\section{Effect of temperature on immature survival}

Temperature significantly affected the mortality recorded over the course of the complete preimaginal period $\left(\chi^{2}\right.$ $=384.4 ; \mathrm{df}=9 ; \mathrm{P}<0.0001)$. This included the mortality recorded for the egg stage and first and second nymphal instars $\left(\chi^{2}=267.7 ; \mathrm{df}=9 ; \mathrm{P}<0.0001\right.$, for the egg stage; $\chi^{2}=242.7 ; \mathrm{df}=9 ; \mathrm{P}<0.0001$, for nymph $1 ; \chi^{2}=139.1$; $\mathrm{df}=8 ; \mathrm{P}<0.0001$, for nymph 2 ), whereas no significant effect was recorded for the third, fourth and fifth nymphal instars, for which mortality ranged between $0 \%$ and $6.7 \%$ $\left(\chi^{2}=10.6 ; \mathrm{df}=7 ; \mathrm{P}=0.1553\right.$ for nymph $3 ; \chi^{2}=6.7 ; \mathrm{df}=$ $7 ; \mathrm{P}=0.4647$ for nymph $4 ; \chi^{2}=5.7 ; \mathrm{df}=7 ; \mathrm{P}=0.5761$ for nymph 5). At the extreme temperatures assayed, $16^{\circ} \mathrm{C}$ and $39^{\circ} \mathrm{C}$, mortality was higher during the egg stage, $\approx 65$ and $71 \%$, respectively. At $16^{\circ} \mathrm{C}$, the first nymphal instar did not develop (100\% mortality) and at $39^{\circ} \mathrm{C}$ suffered a high mortality $(\approx 77 \%)$, and at $39^{\circ} \mathrm{C}$ the second nymphal instar also ceased developing (100\% mortality). Accordingly, the mortality model was fitted to the results for the egg stage, first and second nymphal instars and the total mortality recorded for the whole preimaginal period (Table 2, Fig. 2). The coefficients of determination for these models ranged from 0.88 to 0.99 , indicating the suitability of the function used to fit the mortality data. 
TABle 4. Parameter estimates for the temperature-dependent models describing the relationship between developmental rates $(r=1 / d)$ of all immature stages and instars of $M$. unicostata and temperature $\left({ }^{\circ} \mathrm{C}\right)$, and the temperature thresholds, optimal temperatures and thermal constants predicted by these models.

\begin{tabular}{|c|c|c|c|c|c|c|c|c|}
\hline \multirow{2}{*}{ Model } & \multirow{2}{*}{$\begin{array}{l}\text { Para- } \\
\text { meter }\end{array}$} & \multicolumn{7}{|c|}{ Stage / Instar } \\
\hline & & Egg & Nymph 1 & Nymph 2 & Nymph 3 & Nymph 4 & Nymph 5 & Egg to adult \\
\hline \multirow[t]{4}{*}{ Linear } & $a$ & $-0.1214(0.0083)$ & $-0.4777(0.0575)$ & $-0.5905(0.0520)$ & $-0.7154(0.0806)$ & $-0.6056(0.0656)$ & $-0.3705(0.0231)$ & $-0.0655(0.0048)$ \\
\hline & $b$ & $0.00873(0.00032)$ & $0.03341(0.00224)$ & $0.04426(0.00193)$ & $0.04971(0.00299)$ & $0.04320(0.00243)$ & $0.02708(0.00086)$ & $0.00436(0.00019)$ \\
\hline & $L T$ & $13.9085(0.4685)$ & $14.2979(0.8217)$ & $13.3429(0.6150)$ & $14.3911(0.7897)$ & $14.0204(0.7591)$ & $13.6789(0.4367)$ & $15.0043(0.4985)$ \\
\hline & $K$ & $114.5789(4.2573)$ & $29.9319(2.0051)$ & $22.5955(0.9842)$ & $20.1164(1.2082)$ & $23.1511(1.3027)$ & $36.9223(1.1679)$ & $229.2255(9.8014)$ \\
\hline \multirow[t]{8}{*}{ Logan type III } & $\psi$ & $2.9076(63.2610)$ & $0.9536(0.1128)$ & $5.0839(7.9953)$ & $3.8842(8.3366)$ & $6.5854(53.6592)$ & $2.2864(3.5232)$ & $0.1275(0.0246)$ \\
\hline & $D$ & $90.832(1013.349)$ & $15.2049(3.4365)$ & $61.9892(67.9017)$ & $46.5836(75.0460)$ & $73.2760(362.6676)$ & $48.6683(54.3769)$ & $16.7526(4.8481)$ \\
\hline & $\Delta T$ & $4.7055(9.5815)$ & $0.04673(0.0000)$ & $1.2527(0.2263)$ & $1.1541(1.5491)$ & $1.8007(3.1325)$ & $1.6048(1.4437)$ & $0.8639(0.2567)$ \\
\hline & $T_{b}$ & $7.6260(5.1206)$ & $12.4824(1.4075)$ & $4.4350(4.5055)$ & $6.8115(6.6380)$ & $4.9048(11.3576)$ & $6.0484(4.2777)$ & $12.5227(1.5796)$ \\
\hline & $T_{m}$ & $45.0112(115.6136)$ & $26.5866(0.0000)$ & $36.3670(6.5384)$ & $33.3186(11.5238)$ & $37.8971(31.8448)$ & 35.5928 (9.1953) & $26.7682(1.7193)$ \\
\hline & $L T$ & 8.4 & 12.5 & 4.4 & 6.8 & 4.9 & 6.0 & 12.5 \\
\hline & $U T$ & 43.2 & 39.1 & 39.0 & 38.9 & 39.8 & 39.9 & 39.0 \\
\hline & $O T$ & 35.6 & 38.7 & 35.4 & 35.4 & 35.2 & 35.5 & 35.8 \\
\hline \multirow[t]{7}{*}{ Lactin } & $\rho$ & $0.1174(0.0135)$ & $0.0211(0.0015)$ & $0.0240(0.0007)$ & $0.0254(0.0014)$ & $0.0235(0.0017)$ & $0.0175(0.0006)$ & $0.0039(0.0002)$ \\
\hline & $T_{\max }$ & $44.0589(0.9934)$ & $42.9624(1.3386)$ & $39.9581(0.0920)$ & $39.8730(1.5582)$ & $40.9184(2.3516)$ & $41.3724(1.3651)$ & $41.9287(0.3638)$ \\
\hline & $\Delta$ & $8.4898(0.9604)$ & $2.2710(0.8185)$ & $1.2799(0.1136)$ & $1.2883(0.7339)$ & $1.6760(1.0706)$ & $1.5998(0.5299)$ & $1.2144(0.1565)$ \\
\hline & $\lambda$ & $-0.0521(0.0244)$ & $-1.3404(0.0579)$ & $-1.3154(0.0351)$ & $-1.3730(0.0700)$ & $-1.3331(0.0768)$ & $-1.2470(0.0233)$ & $-1.0603(0.0050)$ \\
\hline & $L T$ & 11.7 & 13.8 & 11.4 & 12.4 & 12.2 & 12.5 & 14.8 \\
\hline & $U T$ & 43.3 & 41.0 & 39.0 & 39.0 & 39.7 & 39.8 & 39.1 \\
\hline & $O T$ & 35.6 & 35.7 & 35.4 & 35.3 & 35.3 & 35.5 & 35.4 \\
\hline
\end{tabular}

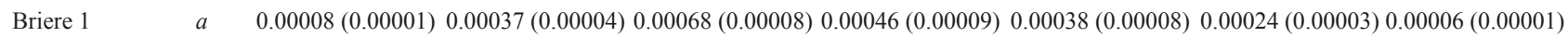

$\begin{array}{llllllll}T_{o}(L T) & 13.4605(0.7817) & 14.7320(1.0351) & 16.1500(1.4150) & 13.8501(1.7869) & 13.0906(1.8855) & 12.7643(1.2947) & 16.3300(0.9901)\end{array}$ $\begin{array}{llllllll}T_{L}(U T) & 42.5321(0.5660) & 41.3737(0.6020) & 39.0000(0.0010) & 42.7417(1.9912) & 43.1001(2.0567) & 43.8271(1.5574) & 39.0000(0.0009)\end{array}$
$O T$
35.7
35.0
33.3
35.9
36.1
36.6
33.4

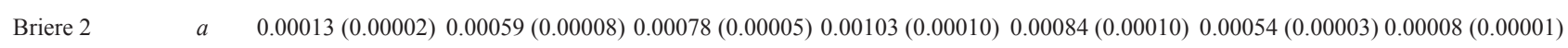

$\begin{array}{llllllll}m & 3.1101(0.8770) & 3.7873(1.3426) & 3.8270(0.7645) & 9.2588(6.2799) & 7.1823(5.5929) & 7.7270(3.0936) & 3.4510(0.5252)\end{array}$

$\begin{array}{llllllll}T_{o}(L T) & 12.2665(1.2702) & 12.9296(1.8797) & 11.6100(2.0989) & 9.7144(3.3951) & 9.4690(4.0329) & 9.0120(1.9948) & 14.0900(1.0695)\end{array}$

$\begin{array}{llllllll}T_{L}(U T) & 40.5461(0.9064) & 39.5268(0.5169) & 39.0000(0.0000) & 37.2523(0.4776) & 37.6129(1.0697) & 37.6517(0.5712) & 39.0000(0.0000)\end{array}$

$\begin{array}{llllllll}O T & 36.0 & 35.8 & 35.3 & 35.6 & 35.5 & 35.7\end{array}$

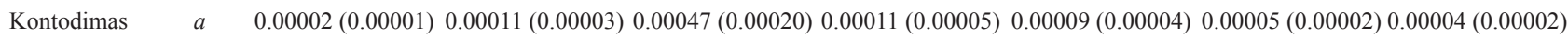
$\begin{array}{llllllll}T_{\min }(L T) & 11.3557(0.9161) & 12.2774(1.4059) & 16.0919(2.2319) & 11.0125(1.9448) & 10.2934(1.9790) & 9.9638(1.3918) & 16.4949(1.9633)\end{array}$ $T_{\max }(U T) \quad 47.7321(1.3414) \quad 45.9299(1.6853) \quad 39.9683(0.8901) \quad 49.6179(4.1914) \quad 50.4243(4.3734) \quad 51.6472(3.3766) \quad 39.9269(0.7832)$
34.7
$32.0 \quad 36.7$
37.0
37.8
32.1

max

Hansen

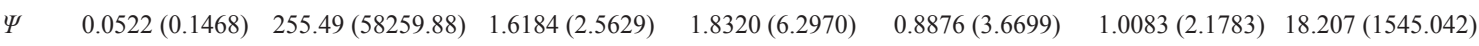

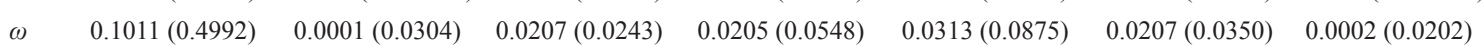

$\begin{array}{llllllll}\Delta_{m} & 7.2067(32.2227) & 1.5004(0.9127) & 1.2604(0.1946) & 1.2128(1.3951) & 1.8240(2.6016) & 1.6808(1.2051) & 1.1835(0.2329)\end{array}$

$\begin{array}{lllllllll}T_{b}(L T) & 8.1502(136.0918) & 14.4560(1.2511) & 11.7918(2.6525) & 12.8842(4.2622) & 11.5796(7.5998) & 12.3564(2.6086) & 14.9717(0.8410)\end{array}$ $\begin{array}{llllllll}T_{m}(U T) & 43.1873(1.5676) & 40.4530(0.7444) & 38.9993(0.0291) & 38.8487(1.7352) & 39.7338(2.7673) & 39.8629(1.5483) & 39.0013(0.0400)\end{array}$
of
$35.6 \quad 36.2$
35.4
35.4
35.2
35.5
35.4

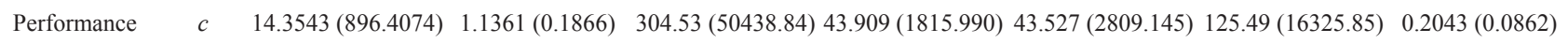

$\begin{array}{llllllll}K_{1} & 0.00062(0.03912) & 0.04451(0.01122) & 0.00015(0.02466) & 0.00113(0.04744) & 0.00099(0.06455) & 0.00022(0.02840) & 0.02706(0.01460)\end{array}$

$\begin{array}{lllllllll}K_{2} & 0.3643(0.3224) & 2.4544(7.7141) & 0.8345(0.1562) & 1.0383(1.5733) & 0.7374(1.3137) & 0.7955(0.7412) & 1.0069(0.1878)\end{array}$

$\begin{array}{lllllllll}T_{1}(L T) & 14.0145(1.2515) & 15.6541(0.3530) & 13.5447(1.8348) & 14.3323(2.7246) & 13.9241(3.6036) & 13.7425(1.6906) & 15.6659(0.4622)\end{array}$

$\begin{array}{llllllll}T_{2}(U T) & 42.0792(1.7234) & 39.4656(1.4461) & 38.9991(0.0300) & 38.5554(1.9780) & 39.2760(3.1441) & 39.2951(1.7097) & 39.0001(0.0275)\end{array}$

$$
\begin{array}{llllllll}
O T & 36.0 & 37.6 & 35.5 & 35.5 & 35.4 & 35.6
\end{array}
$$

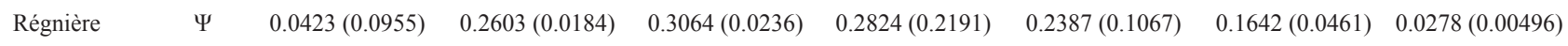

$\begin{array}{lllllllll}\omega & 0.0965(0.3531) & 0.0502(0.0043) & 0.0704(0.0045) & 0.0689(0.0761) & 0.0794(0.0647) & 0.0838(0.0181) & 0.0603(0.0111)\end{array}$

$\begin{array}{llllllll}\Delta_{b} & 1.17 \mathrm{e}+21(0.0000) & 0.2503(0.0308) & 0.0820(0.1079) & 0.3800(1.8272) & 0.1742(0.9719) & 0.0811(0.3280) & 0.3115(0.1147)\end{array}$

$\begin{array}{llllllll}\Delta_{m} & 7.2400(18.8169) & 1.1460(0.2094) & 1.5424(0.1241) & 1.5604(2.7718) & 2.4995(4.3435) & 3.0474(1.6306) & 1.3081(0.2068)\end{array}$

$\begin{array}{lllllllll}T_{b}(L T) & 11.8800(3.5389) & 15.9843(0.1018) & 17.7148(1.4647) & 15.3719(8.6693) & 16.5072(10.0629) & 17.6894(4.6238) & 15.9854(0.2708)\end{array}$

$\begin{array}{llllllll}T_{m}(U T) & 43.1300(1.7207) & 40.0752(0.1628) & 38.9996(0.0172) & 39.0133(2.3196) & 39.9053(2.9327) & 40.5194(1.0789) & 39.0010(0.0269)\end{array}$

$$
\begin{array}{llllllll}
O T & 35.6 & 36.7 & 35.3 & 35.3 & 35.2 & 35.3 & 35.5
\end{array}
$$

$L T$ - predicted lower developmental threshold; $U P$ - predicted upper developmental threshold; $O P$ - optimal temperature for development; $K$ - thermal constant expressed in degree-days (DD); the rest are other parameters of the different temperature-dependent models considered (see the corresponding references for a complete description of the parameters). Values in parentheses are standard errors. 
A

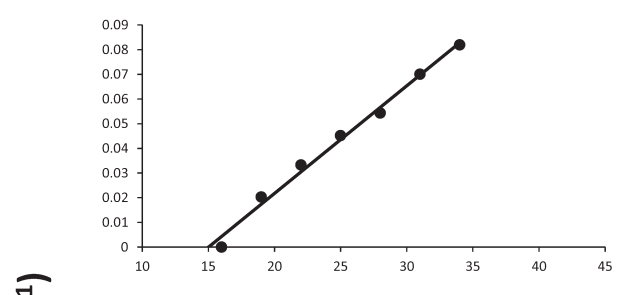

D
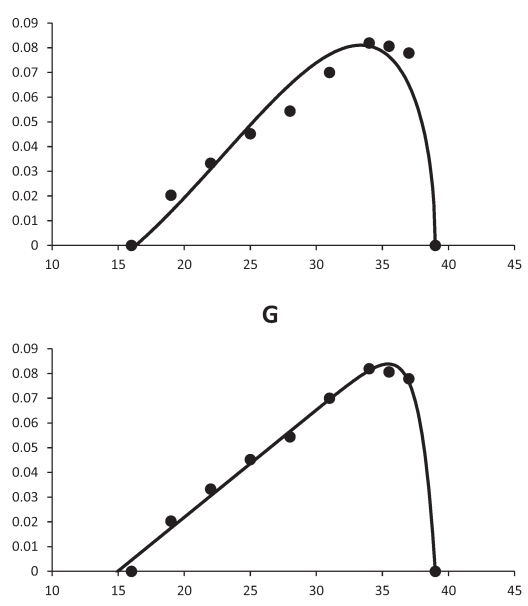

B

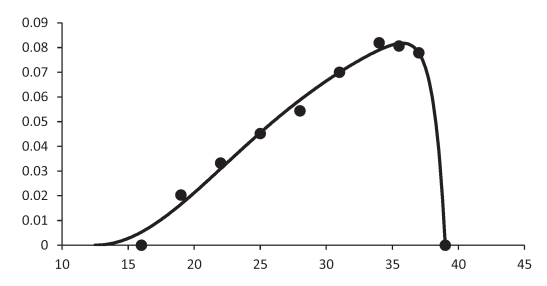

E
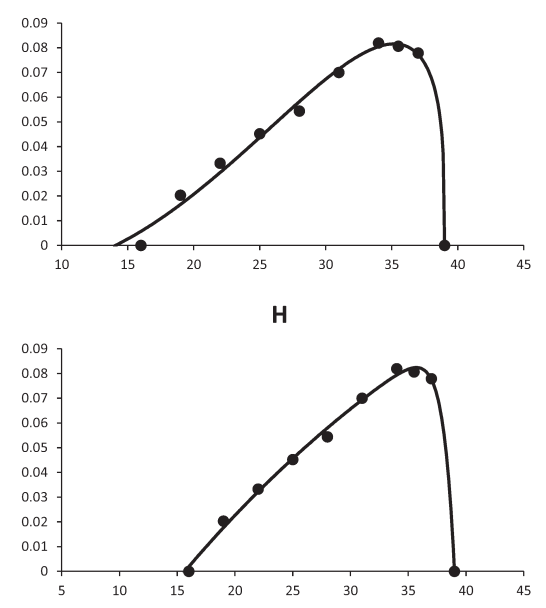

C

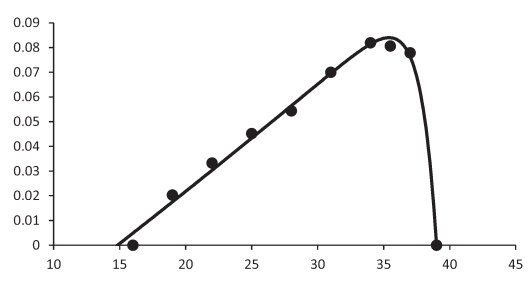

F

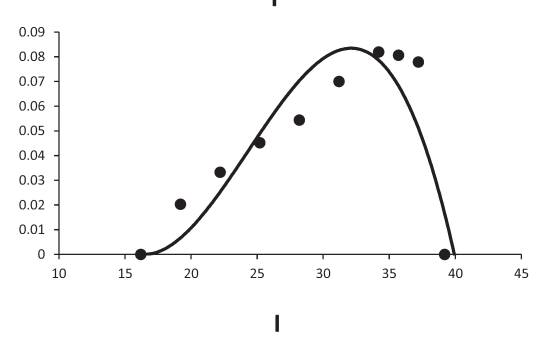

I

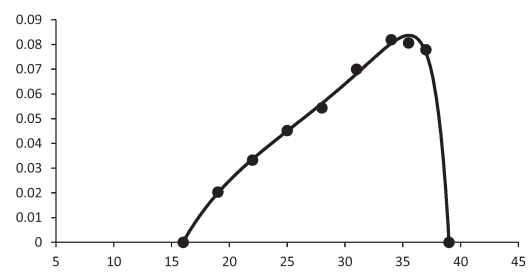

Temperature (으)

Fig. 3. Rates of development $\left(\right.$ day $\left.^{-1}\right)$ for the total preimaginal period at different temperatures $\left({ }^{\circ} \mathrm{C}\right)$ for Monosteira unicostata predicted by the linear (A), Logan III (B), Lactin (C), Briere 1 (D), Briere 2 (E), Kontodimas (F), Hansen (G), Performance (H) and Régnière (I) models. Closed circles are the recorded rates of development and solid lines the fitted curves according to each model.

\section{Effect of temperature on development and thermal requirements of immature stages and instars}

As temperature increased, developmental times decreased with the shortest developmental time recorded at $34-35.5^{\circ} \mathrm{C}$, except for nymph 1 , for which this value was recorded at $37^{\circ} \mathrm{C}$ (Table 3 ). A slight increase was recorded in all cases at temperatures above this optimum. The egg stage took up almost half of the total preimaginal period (45-49\%) and required 114.6 DD (Table 4) for complete development, whereas the second and third nymphal instars had the fastest development, taking up only $8-9 \%$ of this period and required 22.6 and 20.1 DD, respectively, to develop to the next instar. First, fourth and fifth nymphal instars required $11-13 \%$ and 29.9 DD, 9-10\% and 23.2 DD, and $14-15 \%$ and $36.9 \mathrm{DD}$, respectively. The total thermal requirements for the period egg to adult were 229.2 DD. Temperature significantly affected the proportion of time spent in each developmental stage or instar at the different temperatures assayed $(\mathrm{H}=58.9, \mathrm{P}<0.0001$ for the egg; $\mathrm{H}=19.3, \mathrm{P}<0.01$ for nymph $1 ; \mathrm{H}=22.2, \mathrm{P}<0.005$ for nymph $2 ; \mathrm{H}=27.0, \mathrm{P}<0.0005$ for nymph $3 ; \mathrm{H}=44.2$, $\mathrm{P}<0.0001$ for nymph $4 ; \mathrm{H}=36.7, \mathrm{P}<0.0001$ for nymph $5)$. There were no significant differences in the total developmental times recorded for males and females $(\mathrm{F}=0.54$; $\mathrm{df}=1,518 ; \mathrm{P}=0.4633)$. In addition, temperature did not affect the proportions of each sex recorded $\left(\chi^{2}=9.0 ; \mathrm{df}=\right.$
$7 ; \mathrm{P}=0.2494)$ or the expected $1: 1$ sex ratio $\left(\chi^{2}=5.0 ; \mathrm{df}=\right.$ $15 ; \mathrm{P}=0.9924)$.

\section{Models of the developmental rates of the immature stages}

The linear model had high adjusted coefficients of determination $\left(R_{a}^{2}>0.96\right)$ for all the immature stages and instars of $M$. unicostata (Table 5). The lower thermal developmental thresholds predicted by this model were in the range 13.3 to $15.0^{\circ} \mathrm{C}$ (Table 4 ).

The adjusted coefficients of determination for the nonlinear models for all developmental stages and instars were, in general, greater than 0.89 (Table 5), but the graphic representation of the models revealed great differences as can be seen for the complete preimaginal period (Fig. 3 ). When the fitting criteria were considered, it revealed that the Lactin model had the highest $\mathrm{z}$ weights for the complete preimaginal period and for all immature stages and instars except nymph 1 , which was best described by the Performance model (Table 5). The optimal temperature for the development of eggs, the five nymphal instars and the total preimaginal period predicted by the best models ranged between 35.3 and 37.6. In this range, the predicted developmental rates were $0.1741,0.7011,0.9479,0.9993$, $0.8650,0.5636$ and $0.0840 \mathrm{~d}^{-1}$, respectively. In addition, the lower developmental threshold predicted by the best models ranged between 11.4 and $15.7^{\circ} \mathrm{C}$, and the upper threshold ranged between 39.0 and $43.3^{\circ} \mathrm{C}$. 
TABLE 5. Regression analysis of nine models describing the relationship between developmental rates $(r=1 / d)$ and temperature $\left({ }^{\circ} \mathrm{C}\right)$ for all immature stages and instars of M. unicostata.

\begin{tabular}{|c|c|c|c|c|c|c|c|c|}
\hline \multirow{2}{*}{ Model } & \multirow{2}{*}{ Parameter } & \multicolumn{7}{|c|}{ Stage / Instar } \\
\hline & & Egg & Nymph 1 & Nymph 2 & Nymph 3 & Nymph 4 & Nymph 5 & Egg to adult \\
\hline Linear & $R_{a}^{2}$ & 0.9897 & 0.9671 & 0.9874 & 0.9763 & 0.9792 & 0.9934 & 0.9864 \\
\hline \multirow[t]{4}{*}{ Logan type III } & $R_{a}^{2}$ & 0.9866 & 0.9751 & 0.9899 & 0.9577 & 0.9478 & 0.9890 & 0.9814 \\
\hline & $B I C$ & -95.9264 & -61.9689 & -58.1247 & -44.0551 & -44.8661 & -64.1790 & -104.0238 \\
\hline & $A I C c$ & -69.7419 & -35.7845 & -17.3081 & 39.4683 & 38.6573 & 19.3444 & -77.8393 \\
\hline & $z_{i}$ & 0.1494 & 0.0888 & 0.1416 & 0.1057 & 0.0879 & 0.1159 & 0.1294 \\
\hline \multirow[t]{4}{*}{ Lactin } & $R_{a}^{2}$ & 0.9896 & 0.9649 & 0.9934 & 0.9728 & 0.9661 & 0.9936 & 0.9879 \\
\hline & $B I C$ & -98.5588 & -58.5918 & -61.6351 & -46.4245 & -47.1623 & -67.3292 & -108.3732 \\
\hline & $A I C c$ & -85.0717 & -45.1047 & -42.6212 & -16.8217 & -17.5595 & -37.7264 & -94.8861 \\
\hline & $z_{i}$ & 0.2158 & 0.1197 & 0.1643 & 0.2279 & 0.2339 & 0.2362 & 0.1678 \\
\hline \multirow[t]{4}{*}{ Briere 1} & $R_{a}^{2}$ & 0.9837 & 0.9572 & 0.8929 & 0.9495 & 0.9538 & 0.9814 & 0.9316 \\
\hline & $B I C$ & -94.5273 & -57.0812 & -36.6946 & -41.2615 & -44.4486 & -58.6149 & -91.5671 \\
\hline & $A I C c$ & -87.7376 & -50.2915 & -27.4835 & -28.2460 & -31.4330 & -45.5993 & -84.7774 \\
\hline & $z_{i}$ & 0.1460 & 0.1348 & 0.1090 & 0.1357 & 0.1701 & 0.1142 & 0.1116 \\
\hline \multirow[t]{4}{*}{ Briere 2} & $R_{a}^{2}$ & 0.9865 & 0.9621 & 0.9806 & 0.9691 & 0.9615 & 0.9919 & 0.9839 \\
\hline & $B I C$ & -95.9121 & -57.8209 & -51.8894 & -45.4090 & -46.1384 & -65.4877 & -105.5245 \\
\hline & $A I C c$ & -82.4251 & -44.3338 & -32.8755 & -15.8062 & -16.5356 & -35.8849 & -92.0374 \\
\hline & $z_{i}$ & 0.1678 & 0.1090 & 0.1435 & 0.2073 & 0.2025 & 0.2105 & 0.1574 \\
\hline \multirow[t]{4}{*}{ Kontodimas } & $R_{a}^{2}$ & 0.9770 & 0.9364 & 0.5520 & 0.9434 & 0.9480 & 0.9775 & 0.7079 \\
\hline & $B I C$ & -91.0775 & -53.1239 & -23.8157 & -40.3443 & -43.5064 & -57.0700 & -77.0441 \\
\hline & $A I C c$ & -84.2878 & -46.3343 & -14.6046 & -27.3287 & -30.4908 & -44.0545 & -70.2544 \\
\hline & $z_{i}$ & 0.0681 & 0.0703 & 0.0388 & 0.1126 & 0.1389 & 0.0801 & 0.0161 \\
\hline \multirow[t]{4}{*}{ Hansen } & $R_{a}^{2}$ & 0.9870 & 0.9714 & 0.9913 & 0.9593 & 0.9494 & 0.9904 & 0.9863 \\
\hline & $B I C$ & -96.2566 & -60.5484 & -59.4778 & -44.3739 & -45.1203 & -65.2798 & -107.0538 \\
\hline & $A I C c$ & -70.0721 & -34.3639 & -18.6611 & 39.1494 & 38.4031 & 18.2435 & -80.8693 \\
\hline & $z_{i}$ & 0.1554 & 0.0714 & 0.1444 & 0.1129 & 0.0964 & 0.1332 & 0.1405 \\
\hline \multirow[t]{4}{*}{ Performance } & $R_{a}^{2}$ & 0.9788 & 0.9918 & 0.9897 & 0.9558 & 0.9443 & 0.9885 & 0.9914 \\
\hline & $B I C$ & -91.3290 & -73.0598 & -57.9691 & -43.7105 & -44.3452 & -63.8167 & -111.7768 \\
\hline & $A I C c$ & -65.1445 & -46.8753 & -17.1524 & 39.8128 & 39.1781 & 19.7067 & -85.5923 \\
\hline & $z_{i}$ & 0.0520 & 0.2070 & 0.1412 & 0.0977 & 0.0703 & 0.1100 & 0.1574 \\
\hline \multirow[t]{4}{*}{ Régnière } & $R_{a}^{2}$ & 0.9813 & 0.9985 & 0.9969 & 0.9227 & 0.9098 & 0.9913 & 0.9935 \\
\hline & $B I C$ & -93.1637 & -90.3029 & -70.3491 & -42.7029 & -43.9579 & -69.4838 & -115.0614 \\
\hline & $A I C c$ & -39.2818 & -36.4210 & 40.2704 & -* & -* & -* & -61.1795 \\
\hline & $z_{i}$ & 0.0454 & 0.1991 & 0.1172 & - & - & - & 0.1197 \\
\hline
\end{tabular}

$R_{a}^{2}$ - adjusted coefficient of determination; BIC - Bayesian Information Criterion; AICc - corrected Akaike Information Criterion; $z_{i}-\mathrm{z}$ weight that integrates $R_{a}^{2}$, BIC and $A I C c$. *The $A I C c$ could not be calculated because $N-K-1$ was 0 .

\section{Validation of developmental models}

The complete developmental period from egg to adult predicted by the different models for the three fluctuating temperatures regimes revealed that the Lactin model gave the best results (Table 6). The mean deviation from the recorded values using this model was less than $2.4 \%$. The estimates were particularly good for the regime with the highest temperatures tested, with less than $0.8 \%$ deviation, whereas for the intermediate temperature range the deviation was greatest $(<3.7 \%)$. The mean deviations when the Hansen and Régnière models were used were also smaller than $3 \%$ and those when the Performance, Logan III, Briere 2 and Briere 1 models were used were smaller than
$5.2 \%$. The mean deviations when the linear and Kontodimas models were used were the greatest $(>8 \%)$. In the case of the linear model this was due to the estimate for the highest fluctuating temperature regime, which had a deviation greater than $18 \%$, whereas the deviations for the other two regimes were lower than $2.9 \%$.

\section{Distribution of adult emergence}

The distribution of adult emergence was well described by the equation developed by Stinner et al. (1975), as indicated by the high $\mathrm{R}^{2}$ (0.9581) (Fig. 4). Adult emergence began at the normalized age of $0.813(81.3 \%$ of the mean time taken to complete development) and ceased at the normalized age of 1.247 , $(124.7 \%$ of the mean time taken 


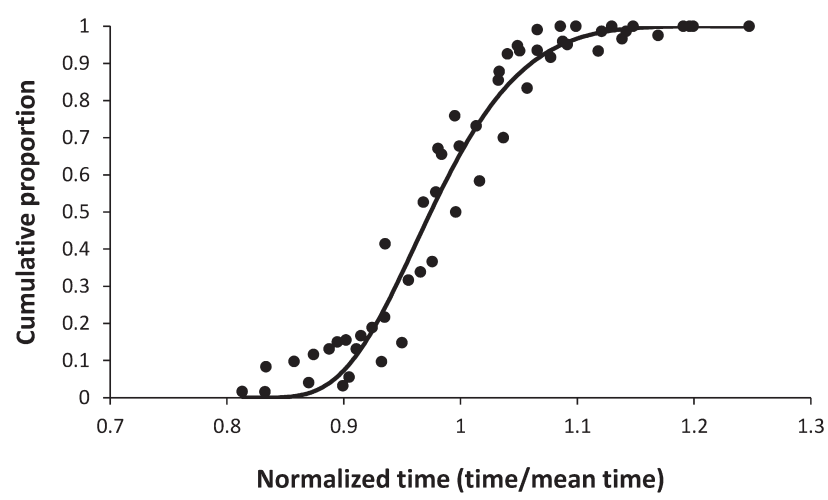

Fig. 4. Cumulative proportion of adult emergence as a function of normalized time (time/mean time) for Monosteira unicostata. Closed circles are the recorded cumulative proportions and solid line the fitted curve according to the Stinner function.

to complete development). The $k$ and $\beta$ parameters were 3.5451 and 3.4870, respectively.

\section{DISCUSSION AND CONCLUSIONS}

Based on the results of our laboratory experiments the poplar lace bug, M. unicostata, can develop over a wide range of temperatures. Within this range, the whole developmental period ranged from 12.1 days to 49.3 days. The values recorded in the field by different authors for the duration of the complete development of M. unicostata range from 20 to 57 days (Bremond, 1938; Vidal, 1939; Gómez-Menor, 1950; Vessia, 1961; Talhouk, 1977; Maniglia, 1983). These values were obtained under the natural fluctuating temperature regimes recorded in the field during spring, summer and early autumn, the seasons when this insect is active. For that reason, the reported durations are highly variable, because it is very likely that the climatic conditions under which they were recorded differed in the different studies. Similarly, these authors also report different development durations for the different genera- tions of this insect depending on the season when they were studied. Although the temperatures during the period when the insect was developing in these field studies were not recorded they nevertheless are similar to our laboratory results obtained under constant and fluctuating temperatures. Our results also indicate the thermophilic nature of this insect reported by different authors (Moleas, 1987; Neal \& Schaefer, 2000), which is in accordance with its geographical distribution around the Mediterranean basin. According to field observations made by Moleas (1987), the optimum temperature for the development of this insect is $30^{\circ} \mathrm{C}$. However, we recorded the shortest developmental time at $34^{\circ} \mathrm{C}$ in our laboratory assays and most of the models indicate the fastest developmental rate occurs at around $35-36^{\circ} \mathrm{C}$.

When the developmental times of M. unicostata are compared with those reported for other tingids, it is amongst those with the fastest development. Thus, other lace bugs like Stephanitis pyrioides (Scott) (Neal \& Douglass, 1988; Braman et al., 1992), S. pyri (Fabricius) (Aysal \& Kivan, 2008), S. takeyai Drake \& Maa (Tsukada, 1994), Corythucha cydoniae (Fitch) (Neal \& Douglass, 1990; Braman \& Pendley, 1993), C. ciliata (Say) (Kim et al., 1999; Ju et al., 2011), C. juglandis (Fitch) (Vogt \& McPherson, 1986), Leptopharsa heveae Drake \& Poor (Cividanes et al., 2004), Leptodictya plana Heidemann (Carr \& Braman, 2012) and Stragulotingis bicincta (Monte) (Ojeda Peña \& Bravo Calderón, 1994) in general take longer to complete their development at temperatures similar to those used in our study. This also indicates that the thermal constant of the poplar lace bug is likely to be one of the lowest for tingids, which is supported by S. takeyai requiring 473.3-479.6 DD to complete its development (Tsukada, 1994); S. pyrioides, 394.0 DD (Braman et al., 1992); S. pyri, 389.6 DD (Aysal \& Kivan, 2008); C. ciliata, 370.6-376.1 DD (Kim et al., 1999; Ju et al., 2011); L. heveae, 370.4 DD (Cividanes et al., 2004) and C. cydoniae, 318.2 DD (Braman \& Pend-

TABLE 6. Total developmental period (days) from egg to adult for $M$. unicostata recorded at three fluctuating temperature regimes and estimates of the models together with percentage deviation $(\% \mathrm{~d})$ of developmental times predicted by each model in relation to the recorded developmental time.

\begin{tabular}{|c|c|c|c|c|c|c|c|}
\hline & \multicolumn{6}{|c|}{ Temperature regime (day : night) } & \multirow{3}{*}{$\begin{array}{c}\text { Mean } \\
\text { deviation }^{b}\end{array}$} \\
\hline & \multicolumn{2}{|c|}{$28-14^{\circ} \mathrm{C}(\mathrm{d}: \mathrm{n})^{a}$} & \multicolumn{2}{|c|}{$33-18^{\circ} \mathrm{C}(\mathrm{d}: \mathrm{n})^{a}$} & \multicolumn{2}{|c|}{$38-22^{\circ} \mathrm{C}(\mathrm{d}: \mathrm{n})^{a}$} & \\
\hline & Days & $\% \mathrm{~d}^{c}$ & Days & $\% \mathrm{~d}^{c}$ & Days & $\% \mathrm{~d}^{c}$ & \\
\hline Experimental data $^{d}$ & $27.14 \pm 0.12$ & - & $17.69 \pm 0.04(698)$ & - & $16.49 \pm 0.05(368)$ & - & - \\
\hline \multicolumn{8}{|l|}{ Model } \\
\hline Linear & 26.36 & -2.87 & 18.20 & 2.88 & 13.46 & -18.37 & $8.04 \pm 5.17$ \\
\hline Logan III & 25.31 & -6.74 & 18.51 & 4.64 & 16.01 & -2.91 & $4.76 \pm 1.11$ \\
\hline Lactin & 26.40 & -2.73 & 18.33 & 3.62 & 16.37 & -0.73 & $2.36 \pm 0.85$ \\
\hline Briere 1 & 23.44 & -13.63 & 17.74 & 0.28 & 16.76 & 1.64 & $5.18 \pm 4.24$ \\
\hline Briere 2 & 25.52 & -5.97 & 18.31 & 3.50 & 15.68 & -4.91 & $4.80 \pm 0.71$ \\
\hline Kontodimas & 22.64 & -16.58 & 18.29 & 3.39 & 18.50 & 12.19 & $10.72 \pm 3.88$ \\
\hline Hansen & 26.37 & -2.84 & 18.35 & 3.73 & 16.34 & -0.91 & $2.49 \pm 0.83$ \\
\hline Performance & 25.64 & -5.53 & 18.47 & 4.41 & 16.05 & -2.67 & $4.20 \pm 0.83$ \\
\hline Régnière & 26.42 & -2.65 & 18.37 & 3.84 & 16.25 & -1.46 & $2.65 \pm 0.69$ \\
\hline
\end{tabular}

${ }^{a} 16 \mathrm{~h}$ day, 8 h night. ${ }^{b}$ Mean \pm SE of the absolute values of the percentages of deviation obtained for each temperature regime. ${ }^{c}$ Negative values indicate an underestimate of the developmental time and positive values indicate an overestimate. ${ }^{d}$ Values are expressed as means \pm standard error. The sample size is indicated within parentheses following each value. 
ley, 1993), compared to the 229.2 DD recorded here for M. unicostata. In contrast, Gargaphia sanchezi Froeschner (Van Schoonhoven et al., 1975) and G. torresi Costa Lima (Da Silva, 2004) take less time to complete their development than $M$. unicostata, with $G$. torresi requiring only 209.4-220.3 DD to reach the adult stage (Da Silva, 2004). Among the lace bugs for which developmental data are available, $C$. morrilli Osborn \& Drake is the most similar in terms of its developmental time to M. unicostata (Rogers, 1977; Stone \& Watterson, 1985). In addition, lack of a difference in the developmental times of males and females is similar to that reported for $S$. pyrioides (Braman et al., 1992) and S. pyri (Aysal \& Kivan, 2008).

During this study of $M$. unicostata we found the published descriptions of nymphal instars to be inaccurate. Similarly, Neal \& Schaefer (2000) point out some discrepancies in the published illustrations of the five nymphal instars of $M$. unicostata. According to our observations (Fig. 1), the drawings of Gómez-Menor (1950) and Vessia (1961) provide an inaccurate description of the different instars. In both cases, the third instar nymph illustrated is a fourth instar nymph and the fourth and fifth instar nymphs are both fifth instar nymphs. In addition, the first and second instar nymphs are also differently illustrated in different publications (Bremond, 1938; Gómez-Menor, 1950; Vessia, 1961; Péricart, 1983). The drawings in Bremond (1938) and Péricart (1983) are most similar to the morphology of the different nymphal instars recorded in our study. However, there is no illustration of the first instar nymph in Péricart (1983) or of the third and fourth instar nymphs in Bremond (1938).

The eggs of $M$. unicostata take longer to hatch than it takes each of the nymphal instars to complete their development. Of the nymphal instars, the second and third instar nymphs had the fastest development, followed by the fourth, first and fifth nymphal instars. This is likely to be a common general pattern among lace bugs because it is similar to that recorded for other tingids (Van Schoonhoven et al., 1975; Vogt \& McPherson, 1986; Neal \& Douglass, 1988, 1990; Braman et al., 1992; Braman \& Pendley, 1993; Ojeda Peña \& Bravo Calderón, 1994; Tsukada, 1994; Kim et al., 1999; Cividanes et al., 2004; Da Silva, 2004; Aysal \& Kivan, 2008; Ju et al., 2011; Carr \& Braman, 2012). In C. morrilli this pattern is somewhat different, although the egg takes the longest to complete development followed by the fifth nymphal instar (Rogers, 1977; Stone \& Watterson, 1985). Despite this general pattern, the proportion of time spent in each developmental stage or instar by M. unicostata was affected by temperature, indicating that in this lace bug the developmental rate isomorphy rule (Jarošík et al., 2002) does not apply.

The lower thermal threshold obtained for the complete preimaginal period of $M$. unicostata is similar to those reported for G. torresi $\left(13.6-13.9^{\circ} \mathrm{C}\right)$ (Da Silva, 2004) and C. cydoniae $\left(14.3^{\circ} \mathrm{C}\right)$ (Braman \& Pendley, 1993) and 3-5 degrees higher than those reported for S. takeyai $\left(\approx 9^{\circ} \mathrm{C}\right)$ (Tsukada, 1994), S. pyrioides $\left(11.2^{\circ} \mathrm{C}\right)$ (Braman et al., 1992), S. pyri $\left(\approx 10^{\circ} \mathrm{C}\right.$ ) (Aysal \& Kivan, 2008), C. ciliata
(11.1-11.2 $\left.{ }^{\circ} \mathrm{C}\right)(\mathrm{Kim}$ et al., 1999; Ju et al., 2011) and L. heveae $\left(9.8^{\circ} \mathrm{C}\right)$ (Cividanes et al., 2004). In all these cases, the lower threshold was estimated using the linear degree-day model, which in our case provided a very similar value to that predicted by the best fitting nonlinear model $\left(15^{\circ} \mathrm{C}\right.$ for the linear model vs. $14.8^{\circ} \mathrm{C}$ for the Lactin model). These differences reflect the different thermal tolerances of these species, with M. unicostata, G. torresi and C. cydoniae better adapted to high temperatures than the other species. However, in the absence of estimates of the optimal temperatures for development and the upper thermal thresholds for the species cited above we are unable to check this. In any case, for some of these species, like $S$. pyrioides, $S$. pyri and $C$. ciliata, no development or very high mortality is recorded at high temperatures ranging from $32^{\circ} \mathrm{C}$ to $36^{\circ} \mathrm{C}$ (Braman et al., 1992; Kim et al., 1999; Aysal \& Kivan, 2008; Ju et al., 2011) while $C$. cydoniae can develop at $33^{\circ} \mathrm{C}$ (Braman \& Pendley, 1993) and here we report development at $37^{\circ} \mathrm{C}$ for $M$. unicostata.

The linear model is widely used to describe the relationship between developmental rate and temperature because of its simplicity and easiness to calculate and apply in practice (Wagner et al., 1984a; Moore \& Remais, 2014). However, its major disadvantage is that it only describes the relationship over the intermediate range of temperatures at which insects can develop. This produces unreal predictions when field temperatures fluctuate outside this range (Wagner et al., 1984a; Moore \& Remais, 2014), a situation that normally occurs within the area of distribution of $M$. unicostata. Our results confirm this, because the prediction provided by the linear model for the highest temperature regime tested resulted in the greatest deviation from the actual duration recorded in this assay, as this regime falls outside the linear portion of the relationship between developmental rate and temperature.

Because of the inaccuracy of the linear model, nonlinear models have been developed to accurately describe the relationship between developmental rate and the whole range of temperatures at which an insect can complete its development. Of the nonlinear models used to fit developmental rate data relative to temperature for $M$. unicostata, the Lactin model was the most accurate. This model has been widely used to describe the relationship between developmental rate and temperature for many insects and mites (Roy et al., 2002; Kontodimas et al., 2004; SánchezRamos et al., 2007; García-Ruiz et al., 2011). In the case of M. unicostata, the Lactin model predicts a thermal window for the complete developmental period (i.e. the range in temperature between the minimum and maximum rate of development for individuals species) of $20.6^{\circ} \mathrm{C}$, which is in accordance with the theoretical predicted value of $20^{\circ} \mathrm{C}$ (Dixon et al., 2009). Using the durations of the complete preimaginal period recorded in fluctuating temperature regimes the Lactin model again provided the best fit and the lowest mean deviation. Therefore, this model could be used as a phenological model in integrated management of M. unicostata to predict the seasonal occurrence of their different generations in the field. In addition, the probabil- 
ity function for the distribution of adult emergence of $M$. unicostata could be used to determine the proportion of individuals that have completed their development at different times in the field. This has practical implications such as the determination of the best moment to apply control measures to reduce the damage done by this pest and the costs associated with pesticide treatments.

The present study has provided information on the effect of temperature on survival and development of the immature stages and instars of $M$. unicostata. In addition, the comprehensive modelling of biological aspects carried out in this work could be used for managing this pest. Further research should focus on the practical application of these models in the field. The need now is to study the effect of temperature on the reproductive and life table parameters of this insect.

ACKNOWLEDGEMENTS. The authors thank H. Sixto [Department of Silviculture and Management of Forest Systems, Forest Research Centre (CIFOR)], INIA, Madrid, for providing the cuttings of poplar. This study was funded by the Spanish Ministry of Agriculture, Food and Environment research project AEG08021-C4-2.

\section{REFERENCES}

Andrewartha H.G. \& Birch L.C. 1954: The Distribution and Abundance of Animals. University of Chicago Press, Chicago, IL, $782 \mathrm{pp}$.

Angilletta M.J. JR. 2006: Estimating and comparing thermal performance curves. - J. Therm. Biol. 31: 541-545.

AYSAL T. \& KIVAN M. 2008: Development and population growth of Stephanitis pyri (F.) (Heteroptera: Tingidae) at five temperatures. - J. Pest Sci. 81: 135-141.

Braman S.K. \& Pendley A.F. 1993: Temperature, photoperiod, and aggregation effects on development, diapause, reproduction, and survival in Corythuch a cydoniae (Heteroptera: Tingidae). - J. Entomol. Sci. 28: 417-426.

Braman S.K., Pendley A.F., Sparks B. \& Hudson W.G. 1992: Thermal requirements for development, population trends, and parasitism of Azalea Lace Bug (Heteroptera: Tingidae). $-J$. Econ. Entomol. 85: 870-877.

BREMOND P. 1938: Le faux-tigre des arbres fruitiers (Monostira unicostata Muls.) au Maroc. - Rev. Pathol. Vég. Entomol. Agric. Fr. 25: 294-307.

Briere J.-F., Pracros P., Le Roux A.-Y. \& Pierre J.-S. 1999: A novel rate model of temperature-dependent development for arthropods. - Environ. Entomol. 28: 22-29.

Burnham K.P. \& Anderson D.R. 2002: Model Selection and Multimodel Inference. A Practical Information-Theoretic Approach. Springer, New York, NY, 516 pp.

Campbell A., Frazer B.D., Gilbert N., Gutierrez A.P. \& MaCKAUER M. 1974: Temperature requirements of some aphids and their parasites. - J. Appl. Ecol. 11: 431-438.

CARR E.R. \& BRAman S.K. 2012: Phenology, abundance, plant injury and effect of temperature on the development and survival of Leptodictya plana (Hemiptera: Tingidae) on Pennisetum spp. grasses. - J. Entomol. Sci. 47: 131-138.

Cividanes F.J., Fonseca F.S. \& Galli J.C. 2004: Biologia de Leptopharsa heveae Drake \& Poor (Heteroptera: Tingidae) e a relação de suas exigências térmicas com a flutuação populacional em seringueira. - Neotrop. Entomol. 33: 685-691.
DA Silva C.A.D. 2004: Efeitos da temperatura no desenvolvimento, fecundidade e longevidade de Gargaphia torresi Lima (Hemiptera, Tingidae). - Rev. Bras. Entomol. 48: 547-552.

De Clerce P. \& Degheele D. 1992: Development and survival of Podisus maculiventris (Say) and Podisus sagitta (Fab.) (Heteroptera: Pentatomidae) at various constant temperatures. Can. Entomol. 124: 125-133.

Dixon A.F.G., HoněK A., Keil P., Kotela M.A.A., Šrzling A.L. \& JAROŠíK V. 2009: Relationship between the minimum and maximum temperature thresholds for development in insects. - Funct. Ecol. 23: 257-264.

García Marí F. \& Ferragut F. 2002: Las Plagas Agrícolas. MV Phytoma-España SL, Valencia, $400 \mathrm{pp}$.

García-Ruiz E., Marco V. \& Pérez-Moreno I. 2011: Effects of variable and constant temperatures on the embryonic development and survival of a new grape pest, Xylotrechus arvicola (Coleoptera: Cerambycidae). — Environ. Entomol. 40: 939947.

GómeZ-Menor J. 1950: La chincheta del almendro (Monostira unicostata Mulsant). - Bol. Patol. Veg. Entomol. Agric. 17: 97-110.

Hansen E.M., Bentz B.J., Powell J.A., Gray D.R. \& VandygrifF J.C. 2011: Prepupal diapause and instar IV developmental rates of the spruce beetle, Dendroctonus rufipennis (Coleoptera: Curculionidae, Scolytinae). - J. Insect Physiol. 57: 1347-1357.

Hilbert D.W. \& Logan J.A. 1983: Empirical model of nymphal development for the migratory grasshopper, Melanoplus sanguinipes (Orthoptera: Acrididae). - Environ. Entomol. 12: $1-5$.

Huey R.B. \& Stevenson R.D. 1979: Integrating thermal physiology and ecology of ectotherms: a discussion of approaches. Am. Zool. 19: 357-366.

Hurvich C.M. \& Tsai C.L. 1989: Regression and time series model selection in small samples. - Biometrika 76: 297-307.

JARoší V., HonĚK A. \& Dixon A.F.G. 2002: Developmental rate isomorphy in insects and mites. - Am. Nat. 160: 497-510.

Ju R.-T., WANG F. \& Li B. 2011: Effects of temperature on the development and population growth of the sycamore lace bug, Corythucha ciliata. - J. Insect Sci. 11: 16.

Kıм G.-H., Сног M.-H. \& KІм J.-W. 1999: Effects of temperatures on development and reproduction of the sycamore lace bug, Corythucha ciliata (Hemiptera, Tingidae). - Kor. J. Appl. Entomol. 38: 117-121 [in Korean, English abstr.].

Kontodimas D.C., Eliopoulus P.A., Stathas G.J. \& Economou L.P. 2004: Comparative temperature-dependent development of Nephus includens (Kirsch) and Nephus bisignatus (Boheman) (Coleoptera: Coccinellidae) preying on Planococcus citri (Risso) (Homoptera: Pseudococcidae): evaluation of a linear and various nonlinear models using specific criteria. - Environ. Entomol. 33: 1-11.

Kvalseth T.O. 1985: Cautionary note about $\mathrm{R}^{2}$. - Am. Stat. 39: 279-285.

Lactin D.J., Holliday N.J., Johnson D.L. \& Craigen R. 1995: Improved rate model of temperature-dependent development by arthropods. - Environ. Entomol. 24: 68-75.

LiotTA G. \& Maniglia G. 1994: Variations in infestations of the almond tree in Sicily in the last fifty years. - Acta Hortic. 373: 277-285.

LiU S.-S., ZhANG G.-M. \& ZHU J. 1995: Influence of temperature variations on rate of development in insects: analysis of case studies from entomological literature. - Ann. Entomol. Soc. Am. 88: 107-119.

LogAn J.A. 1988: Toward an expert system for development of pest simulation models. — Environ. Entomol. 17: 359-376. 
Maniglia G. 1983: Osservazioni biologiche sulla Monosteira unicostata Muls. et Rey (Rhynchota, Tingidae) in Sicilia. Phytophaga 1: 27-40.

MolEAS T. 1987: Etologia, ecologia e controllo della Monosteira unicostata Muls. et Rey sul mandorlo in Puglia. - Difesa Piante 10: 469-483.

Moore J.L. \& Remais J.V. 2014: Developmental models for estimating ecological responses to environmental variability: structural, parametric, and experimental issues. - Acta Biotheor. 62: 69-90.

Moutous G. \& Fos A. 1973: Essais de rhizogénèse chez la feuille de vigne isolée. - Rev. Zool. Agric. Pathol. Vég. 1973(1): $27-32$.

Neal J.W. Jr. \& Douglass L.W. 1988: Development, oviposition rate, longevity, and voltinism of Stephanitis pyrioides (Heteroptera: Tingidae), an adventive pest of azalea, at three temperatures. - Environ. Entomol. 17: 827-831.

Neal J.W. JR. \& Douglass L.W. 1990: Seasonal dynamics and the effect of temperature in Corythuch a cydoniae (Heteroptera: Tingidae). - Environ. Entomol. 19: 1299-1304.

Neal J.W. JR. \& Schaefer C.W. 2000: Lace bugs (Tingidae). In Schaefer C.W. \& Panizzi A.R. (eds): Heteroptera of Economic Importance. CRC Press, Boca Raton, FL, pp. 85-138.

Ojeda Peña D. \& Bravo Calderón M. 1994: Biología y control de Pleseobyrsa bicincta Monte (Hem.: Tingidae), plaga importante de palma aceitera en el Perú. — Oléagineux 49: 145-154.

Péricart J. 1983: Faune de France. 69. Hémiptères Tingidae Euro-méditerranéens. Fédération Française des Société de Sciences Naturelles, Paris, $618 \mathrm{pp}$.

Régnière J. \& Powell J.A. 2013: Animal life cycle models (Poikilotherms). In Schwarz M.D. (ed.): Phenology: An Integrative Environmental Science. Springer, Dordrecht, pp. 295-315.

Régnière J., Powell J., Bentz B. \& Nealis V. 2012: Effects of temperature on development, survival and reproduction of insects: Experimental design, data analysis and modeling. $-J$. Insect Physiol. 58: 634-647.

Rogers C.E. 1977: Laboratory biology of a lace bug on sunflower. - Ann. Entomol. Soc. Am. 70: 144-145.

Sánchez-Ramos I., Álvarez-Alfageme F. \& Castañera P. 2007: Development and survival of the cheese mites, Acarus farris and Tyrophagus neiswanderi (Acari: Acaridae), at constant temperatures and 90\% relative humidity. $-J$. Stor. Prod. Res. 43: 64-72.

Schwarz G. 1978: Estimating dimension of a model. - Ann. Stat 6: $461-464$

SCUdDER G. 2012: Monosteira unicostata (Mulsant \& Rey) (Hemiptera: Tingidae) established in North America, with a key to the genera of Tingidae in Canada. - Entomol. Am. 118: 295-297.
SHI P. \& GE F. 2010: A comparison of different thermal performance functions describing temperature-dependent development rates. - J. Therm. Biology 35: 225-231.

Shi P., Ge F., Sun Y. \& Chen C. 2011: A simple model for describing the effect of temperature on insect developmental rate. $-J$. Asia Pac. Entomol. 14: 15-20.

StatPoint Technologies 2009: Statgraphics ${ }^{\circledR}$ Centurion XVI User Manual. Statpoint Technologies, Warrenton, VA, 305 pp.

Stinner R.E., Butler G.D. JR., Bacheler J.S. \& Tuttle C. 1975: Simulation of temperature-dependent development in population dynamics models. - Can. Entomol. 107: 1167-1174.

Stone J.D. \& WatTerson G.P. 1985: Effects of temperature on the survival and development of the morrill lace bug (Heteroptera: Tingidae) on Guayule. - Environ. Entomol. 14: 329-331.

SYSTAT 2002: TableCurve 2D 5.01 for Windows User's Manual. SYSTAT Software Inc, Richmond, CA, 672 pp.

TALHouk A.S. 1977: Contribution to the knowledge of almond pests in East Mediterranean countries. VI. The sap-sucking pests. - Z. Angew. Entomol. 83: 248-257.

Trudgill D.L., Honek A., Li D. \& Van Straalen N.M. 2005 : Thermal time - concepts and utility. - Ann. Appl. Biol. 146: $1-14$.

TSUKADA M. 1994: The effect of temperature on the development and longevity of the andromeda lace bug, Stephanitis takeyai (Heteroptera: Tingidae) on its two main host plants, Pieris japonica and Lyonia elliptica. - Appl. Entomol. Zool. 29: 571-576.

Van Schoonhoven A., Burbano F. \& Arenas R. 1975: Notes on the biology of the lace bug Gargaphia sanchezi (Hemiptera, Tingidae) a pest of beans (Phaseolus vulgaris). - Turrialba 25: 237.

VESSIA R. 1961: Studio morfo-biologico della Monosteira unicostata Muls. et Rey (Hemiptera - Heteroptera - Tingidae) e difesa del mandorlo. - Ann. Sper. Agrar. (N.S. Suppl.) 15: 1-48.

VIDAL J.P. 1939: Le faux tigre du poirier (Monosteira unicostata Mls. Hem. Heter.). - Bull. Soc. Hist. Nat. Afr. N. 30: 329-336.

Vogt T.E. \& McPherson J.E. 1986: Life history and laboratory rearing of Corythuca juglandis (Hemiptera: Tingidae) with descriptions of immature stages. - Great Lakes Entomol. 19: 221-233.

Wagner T.L., Wu H., Sharpe P.J.H., Schoolfield R.M. \& CoulSON R.N. 1984a: Modeling insect development rates: a literature review and application of a biophysical model. - Ann. Entomol. Soc. Am. 77: 208-225.

Wagner T.L., Wu H.I., Sharpe P.J.H. \& Coulson R.N. 1984b: Modeling distributions of insect development time: a literature review and application of the Weibull function. - Ann. Entomol. Soc. Am. 77: 475-487.

Received March 28, 2015; revised and accepted June 10, 2015 Prepublished online August 27, 2015 